\title{
CURRENT STATUS OF VIRTUAL ACCELERATOR AT J-PARC 3 GEV RAPID CYCLING SYNCHROTRON
}

\author{
H. Harada ${ }^{\#}$, K. Shigaki, Hiroshima University, Hiroshima, Japan \\ F. Noda, H. Hotchi, H. Sako, H. Suzuki, Y. Irie, JAEA, Tokai, Japan \\ K. Furukawa, KEK, Tsukuba, Japan \\ S. Machida, STFC, RAL, Chilton, UK
}

\begin{abstract}
We have developed a Virtual Accelerator based on Experimental Physics and Industrial Control System (EPICS) for $3 \mathrm{GeV}$ Rapid Cycling Synchrotron (RCS) in Japan Proton Accelerator Research Complex (J-PARC). It has a simulator of a beam for a complex accelerator and is put under the control server in parallel with the real machine. It is possible to make a dryrun without a beam, and consequently the Virtual Accelerator gives a strong feedback for the RCS operation as a commissioning tool. In that way, we can examine the control system of the real machine and practice the commissioning of the real machine by using the Virtual Accelerator. It is important to have the tool based on beam dynamics with realistic machine parameters to increase operational performance. We have constructed the Virtual Accelerator system based on EPICS and acceleration process has been implemented into the Virtual Accelerator. A simulation of multi-particles tracking, which we call a dryrun, has been performed on the Virtual Accelerator. Beam energy, synchrotron oscillations and adiabatic damping for horizontal and vertical have been checked out of the dryrun using the Virtual Accelerator. We present the concept of the Virtual Accelerator system and current status of the development.
\end{abstract}

\section{INTRODUCTION}

J-PARC [1] is a high intensity proton machine complex at Tokai in Japan and consists of three parts: $400 \mathrm{MeV}$ (181 MeV at phase 1) LINAC, $3 \mathrm{GeV}$ RCS and $50 \mathrm{GeV}$ (30 GeV at phase 1) Main Ring (MR). In a high intensity machine, an acceptable beam loss rate is lower than that of an usual accelerator. In particular, in the case of RCS, the total acceptable beam loss rate is less than $10^{-3}(1 \mathrm{~kW})$, where RCS has many sources of beam loss caused by space charge effect, nonlinear field components from large aperture magnets and interference of fringe fields etc. If beam loss exceeds $1 \mathrm{~W} / \mathrm{m}$ except for the collimator region, it is impossible to do maintenance of activated elements in the ring. Therefore, the parameters which would cause the excessively beam loss should not be given to the machine modules. So, we have developed a Virtual Accelerator based on EPICS [2] at RCS and the Virtual Accelerator can check the parameters beforehand whether they are usable.

\# harada@hepl.hiroshima-u.ac.jp

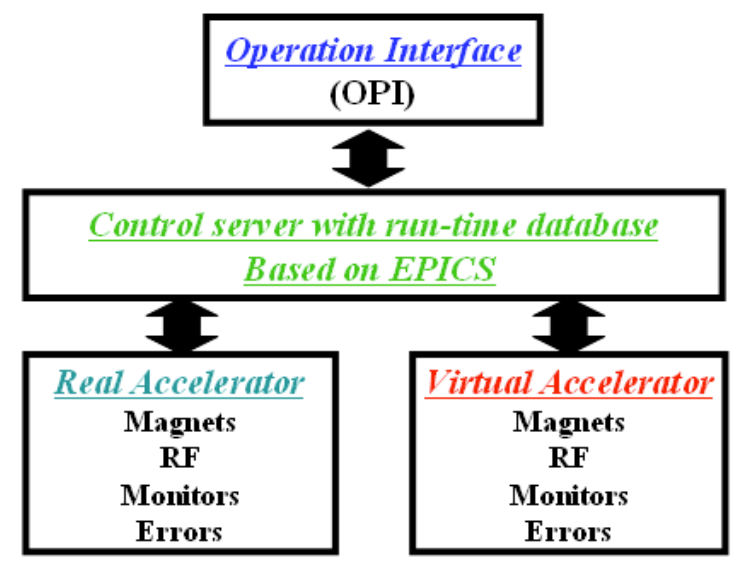

Figure 1: General layout of the VA system based on EPICS.

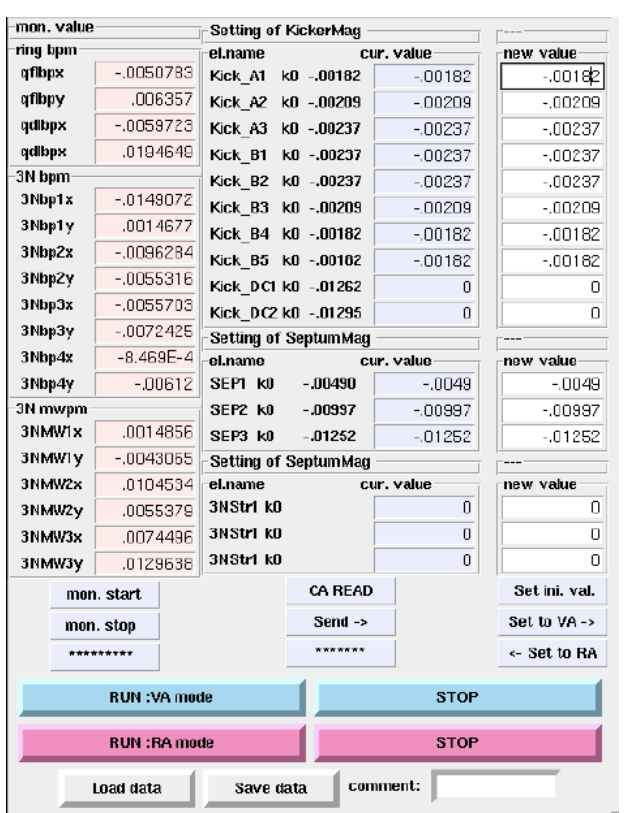

Figure2 : Operation Interface at RCS extraction line.

\section{CONCEPT OF VIRTUAL ACCELERATOR}

Development of the logical accelerator called "Virtual Accelerator" (VA) system allows a revolutionary commissioning and operation of an accelerator. The VA system is able to include various simulation codes based on beam dynamics. In the case of a control system based on EPICS there is an elegant solution facilitating the VA. The VA is put under the control server based on EPICS in parallel with the real machine. This is an originality

T05 Beam Feedback Systems 
control model for an accelerator. This VA system is expected to identify a suitable parameter set of all elements in the real accelerator from a dryrun. Fig.1 shows the general layout of the present VA system.

The control server has a run-time database to the channels identified EPICS records according to operation needs and operators put or get a value into the records by using Channel Access Client (CA Client). Operation Interface (OPI) with Graphical User Interface is a tool to control the elements by using CA Client through the control server and shown in Fig.2. It is possible for an operator to switch the modes between VA mode (blue button) and Real Accelerator mode (pink button). For an operation of the VA through control server, it needs that VA has CA Client in addition to simulation. It allows an operation as well as the real accelerator through control server with run-time database. In this system, it is possible for an operator to control the VA like the real machine and check a beam behavior from a dryrun with VA.

Advantages of the VA system can be summarized in the following. At first, it is possible to examine the control system of the machine and an operator to practice the commissioning of the real machine without a beam.

Secondly, it is possible for an operator to judge whether setting parameters would be justified or not. And finally, it gives a strong feedback for operation of the real machine through the process mentioned above.

\section{CONSTRUCTION OF VIRTUAL ACCLERATOR}

We had constructed the VA system based on EPICS portable channel access server (PCAS), which is used for building of a control server and has a run-time database to EPICS records. We had reported the status of the VA at EPAC 2006 [3]. In that report, we presented the basic control model for VA based on EPICS PCAS, implementation of some elements into the VA and some results of virtual measurement of basic parameters from a dryrun on the VA. However, we have updated the VA system, which is now based on Input/Output Controller (IOC) as described below.

\section{Upgrade of control server for Virtual Accelerator based on EPICS}

We have reconstructed a control server as IOC for VA instead of PCAS. The EPICS IOC has a run-time database to EPICS record and is constructed as control server of the component as well as PCAS. As the difference between IOC and PCAS, the EPICS records in IOC are defined with a record type and fields and have various functions itself, which are a simple calculation, conversion and alarm etc. For example, we convert from a pattern of magnetic field strength to that of current on IOC. Additionally, operators use applications for communication with various devices in IOC, which are VME, PLC and CAMAC etc. Therefore, IOC is constructed as a control server to control the elements in the real machine.

We have replaced a control server of PCAS with that of IOC as well as that of the real machine and reconstructed input or output parts of elements in the VA. As the result, it is possible to use a control server for the real machine as well as the VA. However, name of the records for the VA needs to differ with that for the real machine though the type and fields defined in the record are same, because it causes to operate not the VA but the real machine. In future, we will construct IOCs of all elements for the VA with progress of the real machine.

\section{Implementation of acceleration process}

In the present VA system, we performed the implementation of acceleration process in RCS from 0.181 to $3.0 \mathrm{GeV}$. So, beam energy or momentum in acceleration process changes with time. It is necessary to add the change of various parameters with the process to the VA in order to simulate the beam behavior more realistic.

We have implemented acceleration process into the VA. The process means the acceleration and deceleration of particles in a beam and the change of magnetic field strength of magnets turn by turn. We have run a simulation of multi-particles tracking on the VA, which is called a dryrun without a beam, and checked beam energy from results of a dryrun. The phase spaces evolutions are shown in Fig.3 in step of 5000 turns.

We have analytically checked adiabatic damping for horizontal and vertical phase space using eqn.1 and found to be consistent with the simulation.

$$
\varepsilon \beta \gamma=\text { const }
$$

where $\varepsilon$ is emittance, $\beta$ and $\gamma$ are Lorenz factors.

We have analytically checked synchrotron oscillation in longitudinal phase space from synchrotron tune and also found to be consistent with the simulation. Checking of adiabatic damping for longitudinal phase space is in progress. This is a remaining task for checking of acceleration process in the VA.

\section{FUTURE WORKS}

We will have the first beam commissioning for RCS in Sep. 2007 and then start to apply a new control model including Virtual Accelerator to the real machine. Actually, we will then use the VA for injection or extraction orbit tuning, dynamic aperture survey for the operating point and eliminating the erroneous input data, etc. The knowledge obtained from the beam commissioning is added into the VA in order to reflect a condition of the real machine.

For the first goal, we have some important works for the VA system, especially for the control system as summarized below. 


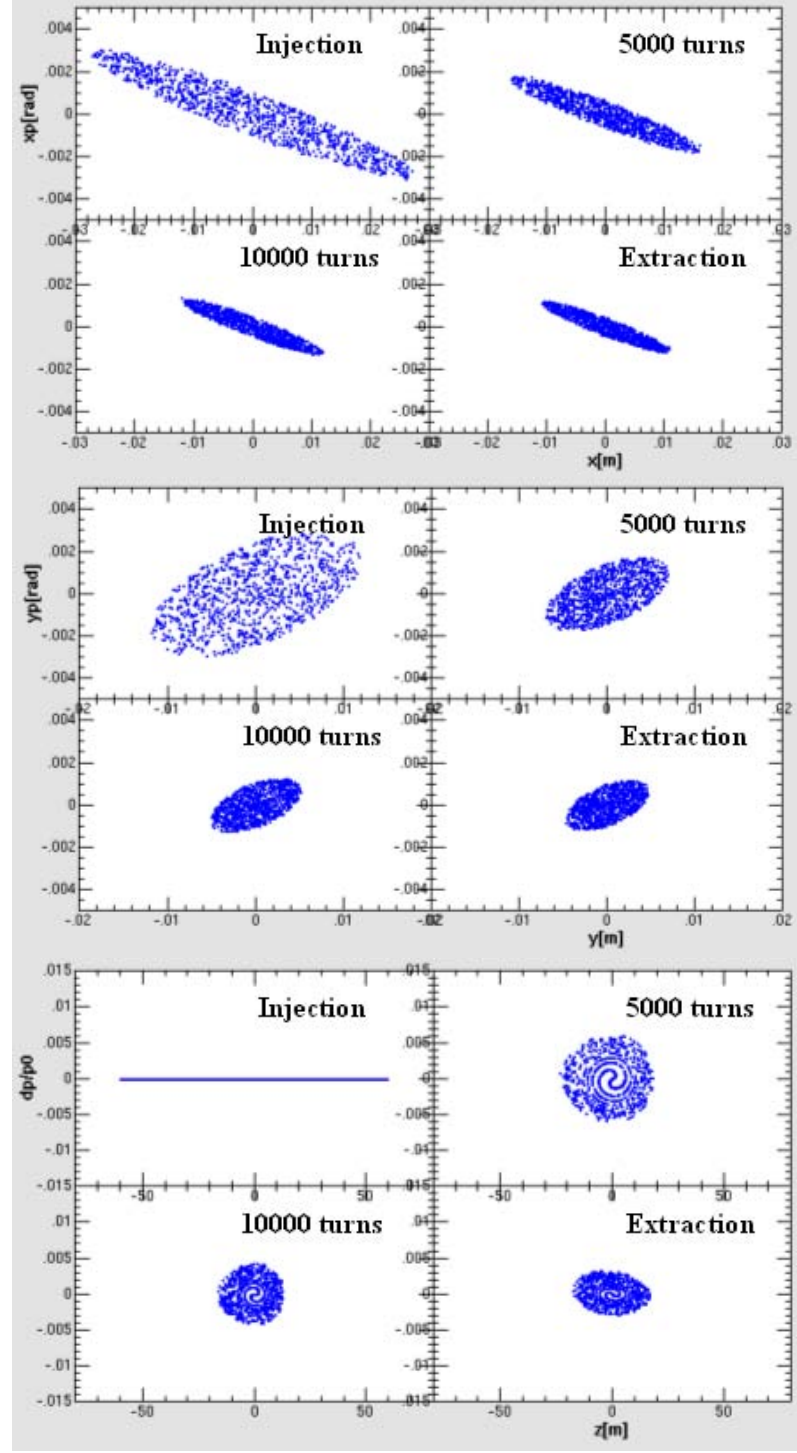

Figure 3 : Horizontal phase $\operatorname{space}\left(\mathrm{x}, \mathrm{x}^{\prime}\right)[\mathrm{up}]$, Vertical phase space $\left(\mathrm{y}, \mathrm{y}^{\prime}\right)$ [middle] and the Longitudinal phase space $(\mathrm{z}, \mathrm{dp} / \mathrm{p} 0)$ [down] from the injection to the extraction with 5000 turns step.

- Construct IOCs of all elements for the VA

- Construct and examine OPI using the VA

- Examine acceleration process

- Do modelling of bump magnets in injection system

- Implement and examine multi-turn injection process

1. We should construct IOCs of all elements for the VA and input or output parts of elements into the VA with progress of the real machine.

2. We needs to construct the operation scheme of the real machine based on a dryrun by the VA. For example, the OPI for commissioning of injection system confirms the injection orbit in physical aperture by a dryrun on the VA and input value of magnets.

3. We have a task for implementation of acceleration process, which is to analytically check adiabatic damping for longitudinal phase space.
4. The magnets with large bore in RCS injection line are aligned very close with each other. It causes magnetic field interference and decrease with each other in the line. Therefore, we need to measure these effects between magnets and put the modelled magnets with these effects on the non-magnet area. Additionally, the magnets should have nonlinear field components. As the result, it is possible to simulate the beam behavior during injection at RCS.

5. Injection scheme at RCS is multi-turns injection with painting injection for 240 turns (24 turns at phase-1) and injection beam is accumulated in the RCS during the time. This process needs to be implemented into the VA. The VA is added a change of field strength of bump magnets and error of injection beam depending on time. As the result, it is possible to examine the injection scheme and commissioning process with the VA.

For the final goal, we have works as summarized below, for operation of RCS with high current or intensity.

Firstly, it is very important to add a calculation with space charge effect in order to estimate a beam loss when operation of high current or intensity. Therefore, we will add the calculation into the VA. Secondly, our goal is to estimate a beam loss for operating parameters online and to limit the operation of an excessive beam loss. However, it takes a few months to run the simulation from injection to extraction. We will try to speed up the simulation by parallelization and simplification.

\section{SUMMARY}

We have developed a Virtual Accelerator system based on EPICS at $3 \mathrm{GeV}$ RCS in J-PARC. This is expected as a tool to give a strong feedback to the real machine and avoid beam loss more than the acceptable one. It is important to have such a tool in a high intensity proton machine like RCS. The Virtual Accelerator built into the same control system in parallel with the real machine makes it possible to make a dryrun without a beam and examine the operation or commissioning algorithm before it is actually executed in the real machine.

We have replaced a control server of PCAS to that of IOC as well as that of the real machine and reconstructed input or output parts of elements in the VA. As a result, it is possible to use the same control server both for the real machine and for the VA. Acceleration Process has been implemented into the VA. A simulation of multi-particles tracking, which we call a dryrun, has been run on the VA. The beam energy, synchrotron oscillations and adiabatic damping for horizontal and vertical have been analytically checked out of the dryrun using the VA.

\section{REFERENCES}

[1] J-PARC, http://jkj.tokai-sc.jaea.go.jp/index-e.html

[2] EPICS, http://www.aps.anl.gov/epics

[3] "Virtual Accelerator as an Operation Tool at J-PARC $3 \mathrm{GeV}$ Rapid cycling Synchrotron (RCS)", H. Harada et al, Proceedings, EPAC, 2006, 